\title{
Scrolls over four dimensional varieties
}

\author{
Andrea Luigi Tironi \\ Dedicated to Prof. A. Lanteri on his 60th birthday \\ (Communicated by A. J. Sommese)
}

\begin{abstract}
We point out the relations between the classical and the adjunction-theoretic definition of scroll over varieties of dimension four. In particular, we prove that an adjunction-theoretic scroll of dimension greater than or equal to seven, polarized by a very ample line bundle, is also a classical scroll and that a classical scroll is an adjunction-theoretic scroll with a few exceptions.
\end{abstract}

Key words. Adjunction theory, adjoint vector bundles, Fano-Mori contraction, extremal ray.

2000 Mathematics Subject Classification. Primary 14N30, 14J40; Secondary 14J60, 14E30, 14C20

\section{Introduction}

Let $X$ be a smooth connected $n$-dimensional variety and let $L$ be an ample line bundle on $X$. In very classical times, the word "scroll", here referred to as a classical scroll, was used to denote a $\mathbb{P}^{k}$-bundle $X$ over a variety $Y$ together with $L$ such that $L_{F} \simeq \mathcal{O}_{\mathbb{P}^{k}}(1)$ for any fiber $F \cong \mathbb{P}^{k}$ with $k=n-\operatorname{dim} Y$. Recently, this definition of scroll has been replaced by another one more adequate from the adjoint theoretic point of view. In modern terms, we say that a pair $(X, L)$ as above is an adjunction-theoretic scroll over a normal variety $Y$, if there exists a morphism with connected fibers, $p: X \rightarrow Y$, such that $K_{X}+(n-\operatorname{dim} Y+1) L \simeq p^{*} H$ for some ample line bundle $H$ on $Y$. The general fiber $F$ of $p$ is such that $\left(F, L_{F}\right) \cong\left(\mathbb{P}^{k}, \mathcal{O}_{\mathbb{P}^{k}}(1)\right), k=n-\operatorname{dim} Y$, but the special fibers can vary quite a lot. So, it seems very natural to investigate the relations between the two definitions of scrolls, by asking the following

Question. What are the differences between classical and adjunction-theoretic scrolls over varieties of small dimension?

In this paper, we give an answer to this Question for scrolls over varieties $Y$ of dimension four, when the polarization $L$ is a very ample line bundle. 
In particular, along one direction, the first two authors of [13] conjectured that an adjunction-theoretic scroll $(X, L)$ over $Y$, with $L$ an ample line bundle on $X$, is also a classical scroll over $Y$ if $n \geq 2 m-1$, where $n=\operatorname{dim} X$ and $m=\operatorname{dim} Y$. Actually, when $L$ is very ample, for $n \geq 2 m+1$ this conjecture is a consequence of a result of Ein (see [14, (1.7)]), for $n=2 m$ it has been considered indirectly in [9], [27] and [32], but for $n=2 m-1$ it remains hard in general. However, the above conjecture was completely solved in the following settings:

(1) $L$ is merely ample and all fibers are $n-m$ dimensional ([15]);

(2) $L$ is ample and spanned and $m \leq 2$ ([28]);

(3) $L$ is very ample and $m=3$ ([13]).

Here we carry on this program by proving that the above conjecture is again true when either $L$ is ample and spanned with $m \leq 3$, or $L$ is very ample and $m=4$ (see Proposition 2.1 and Theorem 2.2 respectively). More precisely, in the latter case, using slicing techniques and results about contractions of smooth variety of dimension four, we are able to determinate in this situation the special fibers of the scroll morphism, $p: X \rightarrow Y$, and to show that these kind of fibers cannot occur.

As to the other direction, in [11] the authors showed that a classical scroll over a smooth manifold $Y$ of dimension $\leq 3$ is an adjunction-theoretic scroll with a few exceptions, relying principally on certain results of ampleness of adjoint bundles to an ample vector bundle together with a part of Mori's theory about extremal rays. In the final section, we make some remarks about classical scrolls which are not adjunction-theoretic scrolls, and we show that a similar result as in $[11,(3.1)]$ can be obtained when $Y$ is a smooth variety of dimension four (see Proposition 3.4) as an immediate consequence of recent classification results about the ampleness of suitable adjoint bundles (see [24] and [4]). Thus, for scrolls over $Y$ with $\operatorname{dim} Y \leq 4$, we have complete results along both directions.

Acknowledgments. Special thanks to Prof. Mauro C. Beltrametti for suggesting the problem and for his guidance throughout this work. The author would like to thank also Prof. Gianluca Occhetta for his kind comments and remarks about the final version of the first part of this paper.

\section{Notation and terminology}

We work over the complex field $\mathbb{C}$. By variety we mean an irreducible and reduced projective scheme $V$ of dimension $n$. We denote its structure sheaf by $\mathcal{O}_{V}$. If $V$ is normal, the dualizing sheaf $K_{V}$ is defined to be $j_{*} K_{\operatorname{Reg}(V)}$, where $j: \operatorname{Reg}(V) \rightarrow V$ is the inclusion of the smooth points of $V$ and $K_{\operatorname{Reg}(V)}$ is the canonical sheaf of holomorphic $n$-forms. Note that $K_{V}$ is a line bundle if $V$ is Gorenstein.

(1.1) We fix some more notation. We denote by

- $\simeq$ the linear equivalence of line bundles;

- $c_{i}(\mathcal{E})$, the $i^{\text {th }}$ Chern class of a vector bundle $\mathcal{E}$ on $V$; 
- $c_{t}(\mathcal{E})=\sum_{i=0}^{r} c_{i}(\mathcal{E}) t^{i}, c(\mathcal{E})=\sum_{i=0}^{r} c_{i}(\mathcal{E})$, the Chern polynomial and the total Chern class of a vector bundle $\mathcal{E}$ on $V$ of rank $r$, respectively;

- $T_{V}$, the tangent bundle of $V$, for $V$ smooth;

- $\mathcal{N}_{U \mid V}$, the normal bundle of $U \subset V$ in $V$.

Line bundles and Cartier divisors are used with little (or no) distinction. Hence we shall freely switch from the multiplicative to the additive notation and vice versa. Sometimes the symbol "." of intersection of cycles is understood.

(1.2) Through this paper it will be assumed that $X$ is a smooth variety ( $n$-fold) of dimension $n \geq 4$.

(1.3) Let $X$ be as in (1.2). A part of Mori's theory of extremal rays is to be used throughout the paper. We will use freely the notation of extremal rays, extremal rational curves and we refer the reader to [19] and [21].

(1.4) Let $X$ be as in (1.2) and let $L$ be an ample line bundle on $X$. We say that $(X, L)$ is an adjunction-theoretic scroll (respectively a quadric fibration, respectively a Del Pezzo fibration, respectively a Mukai fibration) over a normal variety $Y$ of dimension $m$ if there exists a surjective morphism with connected fibers $p: X \rightarrow Y$ and an ample line bundle $H$ on $Y$, such that $K_{X}+(n-m+1) L \simeq p^{*} H$ (respectively $K_{X}+(n-m) L \simeq p^{*} H$, respectively $K_{X}+(n-m-1) L \simeq p^{*} H$, respectively $\left.K_{X}+(n-m-2) L \simeq p^{*} H\right)$. We say that $(X, L)$ is a Del Pezzo variety (respectively a Mukai variety) if $K_{X} \simeq-(n-1) L$ (respectively $\left.K_{X} \simeq-(n-2) L\right)$. A Mukai variety $(X, L)$ is said to be a a ruled Mukai variety of rank $r$ over a smooth variety $Y$ if $(X, L)$ is a Mukai variety and $(X, L) \cong$ $\left(\mathbb{P}_{Y}(\mathcal{E}), \mathcal{O}_{\mathbb{P}(\mathcal{E})}(1)\right)$ for some vector bundle $\mathcal{E}$ of rank $r$ over $Y$. We say that $X$ is a Fano manifold of index $i$ if $-K_{X}$ is ample and $i$ is the largest integer such that $-K_{X} \simeq i H$ for some ample line bundle $H$ on $X$. Finally, we say that $(X, L)$ is a $\mathbb{P}^{k}$-bundle over a smooth variety $Y$, or a classical scroll, if there exists a surjective morphism $p: X \rightarrow Y$ such that all fibers $F$ of $p$ are $\mathbb{P}^{k}$ and $L_{F} \cong \mathcal{O}_{\mathbb{P}^{k}}(1)$. This is equivalent to say that $(X, L) \cong\left(\mathbb{P}_{Y}(\mathcal{E}), \mathcal{O}_{\mathbb{P}(\mathcal{E})}(1)\right)$, where $\mathcal{E}=p_{*} L$ is an ample vector bundle of rank $k+1$ on $Y$. In this case the canonical bundle formula gives $K_{X}+(k+1) L \simeq p^{*}\left(K_{Y} \otimes \operatorname{det} \mathcal{E}\right)$.

(1.5) Finally, for general results on adjunction theory we refer to [28] and [12]. For some further results on scrolls we refer to [10, (0.6), (3.1) and $\S 4]$ and also to $[13, \S 3]$.

\section{Adjunction-theoretic scrolls}

First of all, let us give here a consequence of well-known results.

Proposition 2.1. Let $L$ be an ample and spanned line bundle on an $n$-fold $X$. Assume that $(X, L)$ is an adjunction-theoretic scroll, $p: X \rightarrow Y$, over a normal projective variety $Y$ of dimension $m \leq 3$. If $n \geq 2 m-1$, then $Y$ is smooth and $p$ is a $\mathbb{P}^{d}$-bundle with $d=n-m$.

Proof. If $m=1,2$, then the result follows from [28, (3.3)]. So we assume that $m=3$. By $[13,(3.2 .1)]$ we know that $p: X \rightarrow Y$ has no divisorial fibers. Moreover, from [15, (2.12)] we deduce that $p$ is a $\mathbb{P}^{d}$-bundle with $d=n-m$ unless there exist isolated special 
fibers $F$ of dimension $n-2$. Since

$$
\operatorname{dim} F=n-2=n-3+1=\operatorname{dim} X-\operatorname{dim} Y+1,
$$

by $[6,(4.1)(i i)]$ we conclude that $n-2 \leq \frac{n}{2}$, i.e., $n \leq 4$, but this gives a contradiction.

On the other hand, when the base $Y$ of the scroll projection $p: X \rightarrow Y$ has dimension four, we can give the following extension of [13, (3.2.3)].

Theorem 2.2. Let $L$ be a very ample line bundle on an $n$-fold $X$. Assume that $(X, L)$ is an adjunction-theoretic scroll, $p: X \rightarrow Y$, over a normal projective variety $Y$ of dimension $m \leq 4$. If $n \geq 2 m-1$, then $Y$ is smooth and $p$ is a $\mathbb{P}^{d}$-bundle with $d=n-m$.

Proof. For $m \leq 3$ the result is shown to be true by Proposition 2.1 (see also [13, (3.2.3)]). Moreover, if $n \geq 2 m$, then by [32, (2.6)], we get the statement. So we can assume that $n=7$ and $m=4$. Note that [13, (3.2.1)] applies to say that $p$ is the contraction of a numerically effective extremal ray $R=\mathbb{R}_{+}[l]$, where $l$ is a line in a general fiber $\mathbb{P}^{3}$ and $p$ has no divisorial fibers. Let $\mathcal{Z}:=\left\{y \in Y \mid \operatorname{dimp}^{-1}(y)>3\right\}$. By [13, (3.2.5)] we see that $\mathcal{Z}$ is finite. Note that $p$ has no fibers of dimension four, since otherwise by [6, (4.1)(ii)] it would follow that $4 \leq \frac{n}{2}$, i.e., $n \geq 8$, but this is absurd.

Now, let $F$ be a five dimensional fiber of the scroll projection $p: X \rightarrow Y$.

Claim. The pair $\left(F, L_{F}\right)$ is either (i) $\left(\mathbb{P}^{5}, \mathcal{O}_{\mathbb{P}^{5}}(1)\right)$, or (ii) $\left(\mathbb{Q}^{5}, \mathcal{O}_{\mathbb{Q}^{5}}(1)\right)$, where $\mathbb{Q}^{5} \subset \mathbb{P}^{6}$ is a possibly singular or reducible hyperquadric of $\mathbb{P}^{6}$.

Take a very ample divisor $D$ on $Y$ such that $W_{6}=p^{*} D$ is a smooth 6-fold and let $H_{i} \in|L|$ be general hyperplane sections for $i=1,2,3$, such that

$$
W_{5}=W_{6} \cap H_{1}, \quad W_{4}=W_{6} \cap H_{1} \cap H_{2}, \quad V=X \cap H_{1} \cap H_{2} \cap H_{3}
$$

are smooth and $\operatorname{dim} S=2$ with $S=F \cap V$. Since $K_{X}+4 L \simeq p^{*} H$ for some ample line bundle $H$ on $Y$, by adjunction $\left(W_{6}, L_{W_{6}}\right)$ and $\left(W_{5}, L_{W_{6} \mid W_{5}}\right)=\left(W_{5}, L_{W_{5}}\right)$ are scrolls over a normal 3-fold $Y_{3} \in|D|$. Thus by [13, (3.2.3)] these pairs are actually classical scrolls. Then $\left(W_{4}, L_{W_{5} \mid W_{4}}\right)$ is a scroll with at worst fibers of dimension two. Consider the restriction $p_{V}: V \rightarrow Y$ of $p$ to $V$. By adjunction we have $K_{V}+L_{V} \simeq p_{V}^{*} H$ and since $Y$ is normal and the fibers of $p_{V}$ are connected, $p_{V}$ is the morphism associated to $\left|N\left(K_{V}+L_{V}\right)\right|$ for $N>>0$. Since $V$ is smooth, from [12, (4.2.14)] and [13, (1.3.3)] we deduce that $p_{V}$ is the contraction of the extremal face

$$
\left(K_{V}+L_{V}\right)^{\perp} \cap \overline{\mathrm{NE}}(X)-\{0\},
$$

where " $\perp$ " means the orthogonal complement (see also [19]). Thus from [20, (8-1-3) and (8-1-4)(i)] it follows that $p_{V}$ is an extremal contraction (or a Fano-Mori contraction). Assume that the two dimensional fiber $S$ is not an isolated fiber of $p_{V}$. Then $S$ is contained in a one dimensional family $E$ of surfaces $f$ such that $p_{V}(f)$ is a point and $p_{V}(E)$ is a curve $C$ on $Y$. Note that each surface $f$ comes from a general fiber $\widehat{F}=\mathbb{P}^{3}$ of $p$ and since $L_{\widehat{F}} \simeq \mathcal{O}_{\mathbb{P}^{3}}(1)$, we deduce that $f \cong \mathbb{P}^{2}$ with $L_{f} \simeq \mathcal{O}_{f}(1)$. Then $E$ is a $\mathbb{P}^{2}$-bundle over $C$ and

$$
K_{f}=K_{V \mid f}+c_{1}\left(\mathcal{N}_{f \mid V}\right)=-L_{f}+c_{1}\left(\mathcal{N}_{f \mid V}\right)
$$


i.e., $c_{1}\left(\mathcal{N}_{f \mid V}\right)=\mathcal{O}_{\mathbb{P}^{2}}(-2)$. From the exact sequence

$$
0 \rightarrow \mathcal{N}_{f \mid E} \cong \mathcal{O}_{f} \rightarrow \mathcal{N}_{f \mid V} \rightarrow \mathcal{N}_{E \mid V_{\mid f}} \rightarrow 0,
$$

we get $\mathcal{N}_{E \mid V_{\mid f}}=\mathcal{O}_{\mathbb{P}^{2}}(-2)$ and so $\mathcal{N}_{f \mid V} \cong \mathcal{O}_{\mathbb{P}^{2}} \oplus \mathcal{O}_{\mathbb{P}^{2}}(-2)$. Since $Y_{3} \cap C \neq \emptyset$ and $f \subset V \subset H_{i}$ for $i=1,2,3$, then some $f$ 's are fibers of $W_{4}$. By [13, (3.2.4)] or [12, (14.1.4)] we know that $\mathcal{N}_{f \mid W_{4}} \cong \mathcal{T}_{\mathbb{P}^{2}}(-2)$. Thus from the exact sequence

$$
0 \rightarrow \mathcal{N}_{f \mid V} \rightarrow \mathcal{N}_{f \mid X \cap H_{1} \cap H_{2}} \rightarrow \mathcal{N}_{V\left|X \cap H_{1} \cap H_{2}\right| f} \rightarrow 0,
$$

i.e.,

$$
0 \rightarrow \mathcal{O}_{\mathbb{P}^{2}} \oplus \mathcal{O}_{\mathbb{P}^{2}}(-2) \rightarrow \mathcal{N}_{\mathbb{P}^{2} \mid X \cap H_{1} \cap H_{2}} \rightarrow \mathcal{O}_{\mathbb{P}^{2}}(1) \rightarrow 0,
$$

it follows that $c_{1}\left(\mathcal{N}_{\mathbb{P}^{2} \mid X \cap H_{1} \cap H_{2}}\right)=\mathcal{O}_{\mathbb{P}^{2}}(-1)$ and $c_{2}\left(\mathcal{N}_{\mathbb{P}^{2} \mid X \cap H_{1} \cap H_{2}}\right)=-2$. Since $f \cong$ $\mathbb{P}^{2} \subset W_{4} \subset X \cap H_{1} \cap H_{2}$, from

$$
0 \rightarrow \mathcal{N}_{f \mid W_{4}} \rightarrow \mathcal{N}_{f \mid X \cap H_{1} \cap H_{2}} \rightarrow \mathcal{N}_{W_{4}\left|X \cap H_{1} \cap H_{2}\right| f} \rightarrow 0
$$

that is,

$$
0 \rightarrow \mathcal{T}_{\mathbb{P}^{2}}(-2) \rightarrow \mathcal{N}_{\mathbb{P}^{2} \mid X \cap H_{1} \cap H_{2}} \rightarrow \mathcal{O}_{\mathbb{P}^{2}} \rightarrow 0,
$$

we obtain that $c_{2}\left(\mathcal{N}_{\mathbb{P}^{2} \mid X \cap H_{1} \cap H_{2}}\right)=c_{2}\left(\mathcal{T}_{\mathbb{P}^{2}}(-2)\right)=1$, a contradiction. Thus we can assume that $S$ is an isolated fiber of $p_{V}$. By [8, (4.11)] we get that $\left(S, L_{S}\right)$ is one of the following pairs:

$$
\left(\mathbb{P}^{2}, \mathcal{O}_{\mathbb{P}^{2}}(1)\right), \quad\left(\mathbb{P}^{1} \times \mathbb{P}^{1}, \mathcal{O}_{\mathbb{P}^{1} \times \mathbb{P}^{1}}(1,1)\right), \quad\left(\mathbb{S}_{2}, \mathcal{O}(1)\right), \quad\left(\mathbb{P}^{2} \sqcup \mathbb{P}^{2}, \mathcal{O}(1)\right),
$$

where $\mathbb{S}_{2}$ is the (normal) cone defined by contracting the curve of minimal self-intersection $C_{0}$ on the Hirzebruch surface $\mathbb{F}_{2}$ to a normal point. Then $L_{F}^{5}=L_{S}^{2} \leq 2$ and since $L_{F}$ is very ample, we obtain the claim.

Consider now a limit $P$ of general fibers $\widehat{F}$ of $p$. Since $L$ is very ample and $\widehat{F} \cong \mathbb{P}^{3}$, we see that $P \cong \mathbb{P}^{3}$. Then the fiber $F$ must contain at least a linear space $P \cong \mathbb{P}^{3}$. Since $P \subset X$ is a smooth irreducible subvariety of degree one relative to $L$, from [12, (6.6.1)] we know that $\left|L \otimes \mathcal{I}_{P}\right|$ is spanned by global sections on $X$. So by [12, (1.7.5)] we can take a general hyperplane $H_{1}$ containing $P \cong \mathbb{P}^{3}$ and such that $X \cap H_{1}$ is a smooth 6fold. Take a general hyperplane section $H_{2}$ such that $\mathbb{P}_{a}^{2}=P \cap H_{2}$ and $X \cap H_{1} \cap H_{2}$ is a smooth 5-fold. By arguing as above, we can take an hyperplane $H_{3}$ containing $\mathbb{P}_{a}^{2}$ and such that $V=X \cap H_{1} \cap H_{2} \cap H_{3}$ is a smooth 4-fold with a fiber $S=F \cap V$ given by either $\mathbb{P}_{a}^{2}$ in Case (i) or $\mathbb{P}_{a}^{2} \sqcup \mathbb{P}^{2}$ in Case (ii). Consider a line $l \subset \mathbb{P}_{a}^{2}$ and by [12, (6.4.2)] let $\rho=\operatorname{cont}_{R}: V \rightarrow T$ be the contraction of the extremal ray $R=\mathbb{R}_{+}[l]$ onto a normal 4-fold $T$ such that $p_{V}=\mu \circ \rho$, where $\mu: T \rightarrow Y$ has connected fibers. Let $E$ be the locus of $R$. Note that $\rho\left(\mathbb{P}_{a}^{2}\right)$ is a point and so $\mathbb{P}_{a}^{2} \subset E$. In particular, if $\rho(E)=\rho(S)$, then $E \subseteq S$. By results of [18], [8], [7] (see also [3, (4.1.3)]), shrinking eventually the morphism $\rho$, we obtain the following possibilities:

(1) $\rho$ has signature $(2,0)$ and $E=S=\mathbb{P}_{a}^{2}$ with $\mathcal{N}_{S \mid V} \cong \mathcal{O}_{\mathbb{P}^{2}}(-1)^{\oplus 2}$; 
(2) $\rho$ has signature $(3,1), E$ is an irreducible $\mathbb{P}^{2}$-bundle, $S=\mathbb{P}_{a}^{2}$ and either

(a) $\mathcal{N}_{S \mid V} \cong \mathcal{O}_{\mathbb{P}^{2}}(-1) \oplus \mathcal{O}_{\mathbb{P}^{2}} ;$ or

(b) $\mathcal{N}_{S \mid V} \cong \mathcal{O}_{\mathbb{P}^{2}}(-2) \oplus \mathcal{O}_{\mathbb{P}^{2}}$;

(3) $\rho$ has signature $(3,1), E$ is an irreducible quadric bundle over a curve and $S=\mathbb{P}_{a}^{2} \sqcup \mathbb{P}^{2}$ with $\mathcal{N}_{S \mid V} \cong \mathcal{O}(-1) \oplus \mathcal{O}$;

(4) $\rho$ has signature $(3,2)$ with

(c) $S=\mathbb{P}_{a}^{2}$ and $\mathcal{N}_{S \mid V}^{*} \cong \mathcal{T}_{\mathbb{P}^{2}}(-1) \oplus \mathcal{O}_{\mathbb{P}^{2}}(1) / \mathcal{O}_{\mathbb{P}^{2}}$;

(d) $S=\mathbb{P}_{a}^{2}$ and $\mathcal{N}_{S \mid V}^{*} \cong \mathcal{O}_{\mathbb{P}^{2}}^{\oplus 4} / \mathcal{O}_{\mathbb{P}^{2}}(-1)^{\oplus 2}$;

(e) $S=\mathbb{P}_{a}^{2} \sqcup \mathbb{P}^{2}$ with $\mathcal{N}_{S \mid V}^{*} \cong \mathcal{T}_{\mathbb{P}^{2}}(-1) \sqcup\left(\mathcal{O}_{\mathbb{P}^{2}} \oplus \mathcal{O}_{\mathbb{P}^{2}}(-1)\right)$.

Since by adjunction we have that

$$
\mathcal{O}_{\mathbb{P}_{a}^{2}}(-3)=K_{\mathbb{P}_{a}^{2}}=K_{V \mid \mathbb{P}_{a}^{2}}+c_{1}\left(\mathcal{N}_{\mathbb{P}_{a}^{2} \mid V}\right) \simeq-L_{\mathbb{P}_{a}^{2}}+c_{1}\left(\mathcal{N}_{\mathbb{P}_{a}^{2} \mid V}\right),
$$

i.e., $c_{1}\left(\mathcal{N}_{\mathbb{P}_{a}^{2} \mid V}\right)=\mathcal{O}_{\mathbb{P}_{a}^{2}}(-2)$, it follows that Cases (2)(a), (3) and (4)(e) are not possible. This gives that Case (ii) in the Claim does not occur.

Let us work out Cases (1) and (2)(b). Actually, since $F \cong \mathbb{P}^{5}$ and $\mathcal{N}_{F|X| \mathbb{P}_{a}^{2}} \cong \mathcal{N}_{\mathbb{P}_{a}^{2} \mid V}$, from [25, (2.3.2)] we deduce that $\mathcal{N}_{F \mid X} \cong \mathcal{O}_{\mathbb{P}^{5}}(-1)^{\oplus 2}, \mathcal{O}_{\mathbb{P}^{5}} \oplus \mathcal{O}_{\mathbb{P}^{5}}(-2)$. Therefore, from the exact sequence

$$
0 \rightarrow \mathcal{N}_{P \mid F} \cong \mathcal{O}_{\mathbb{P}^{3}}(1)^{\oplus 2} \rightarrow \mathcal{N}_{P \mid X} \rightarrow \mathcal{N}_{F \mid X} \rightarrow 0,
$$

we see that $c_{2}\left(\mathcal{N}_{P \mid X}\right) \neq 0$, but this gives a contradiction since the normal bundle of $P \cong \mathbb{P}^{3}$ in $X$ is a specialization of the trivial one.

Finally, consider Cases (4)(c) and (4)(d). Put $c_{i}:=c_{i}\left(\mathcal{N}_{\mathbb{P}_{a}^{2} \mid V}\right)$ for $i=1,2$. Then, we have the following possibilities:

- $\left(c_{1}, c_{2}\right)=\left(-2 H, 2 H^{2}\right)$ in Case $(4)(\mathrm{c})$;

- $\left(c_{1}, c_{2}\right)=\left(-2 H, 3 H^{2}\right)$ in Case $(4)(\mathrm{d})$,

where $H$ is the class of a hyperplane in a linear space. Since $\mathbb{P}_{a}^{2} \subset V \subset X \cap H_{1}$, from the following exact sequence

$$
0 \rightarrow \mathcal{N}_{\mathbb{P}_{a}^{2} \mid V} \rightarrow \mathcal{N}_{\mathbb{P}_{a}^{2} \mid X \cap H_{1}} \rightarrow \mathcal{N}_{V \mid X \cap H_{1 \mid \mathbb{P}_{a}^{2}}} \cong \mathcal{O}_{\mathbb{P}_{a}^{2}}(1)^{\oplus 2} \rightarrow 0
$$

we deduce that

$$
c_{i}\left(\mathcal{N}_{\mathbb{P}_{a}^{2} \mid X \cap H_{1}}\right)=0 \quad \text { for } \quad i=1,3,4 \quad \text { and } \quad c_{2}\left(\mathcal{N}_{\mathbb{P}_{a}^{2} \mid X \cap H_{1}}\right)=H^{2}+c_{2}+2 H c_{1} .
$$

Since $\mathbb{P}_{a}^{2} \subset P \subset X \cap H_{1}$, consider the exact sequence

$$
0 \rightarrow \mathcal{N}_{\mathbb{P}_{a}^{2} \mid P} \cong \mathcal{O}_{\mathbb{P}_{a}^{2}}(1) \rightarrow \mathcal{N}_{\mathbb{P}_{a}^{2} \mid X \cap H_{1}} \rightarrow \mathcal{N}_{P\left|X \cap H_{1}\right| \mathbb{P}_{a}^{2}} \rightarrow 0 .
$$

Then we have that

$$
c_{1}\left(\mathcal{N}_{P \mid X \cap H_{1 \mid \mathbb{P}_{a}^{2}}}\right)=c_{1}\left(\mathcal{N}_{P \mid X \cap H_{1}}\right)_{\mid \mathbb{P}_{a}^{2}}=-H
$$

and

$$
c_{2}\left(\mathcal{N}_{P \mid X \cap H_{1 \mid \mathbb{P}_{a}^{2}}}\right)=c_{2}\left(\mathcal{N}_{P \mid X \cap H_{1}}\right)_{\mid \mathbb{P}_{a}^{2}}=2 H^{2}+c_{2}+2 c_{1} H,
$$


and this gives

$$
c_{1}\left(\mathcal{N}_{P \mid X \cap H_{1}}\right)=-H \quad \text { and } \quad c_{2}\left(\mathcal{N}_{P \mid X \cap H_{1}}\right)=2 H^{2}+c_{2}+2 c_{1} H .
$$

Moreover, since $P \subset X \cap H_{1} \subset X$, consider also the following exact sequence

$$
0 \rightarrow \mathcal{N}_{P \mid X \cap H_{1}} \rightarrow \mathcal{N}_{P \mid X} \rightarrow \mathcal{N}_{X \cap H_{1} \mid X} \cong \mathcal{O}_{\mathbb{P}^{3}}(1) \rightarrow 0 .
$$

Since $\mathcal{N}_{P \mid X}$ is a specialization of a trivial bundle, then $c_{i}\left(\mathcal{N}_{P \mid X}\right)=0$ for $i=1,2,3$, and in Case (4)(c) we have a numerical contradiction, while in Case (4)(d) we obtain

$$
c_{3}\left(\mathcal{N}_{P \mid X \cap H_{1}}\right)=-H^{3}, \quad c_{2}\left(\mathcal{N}_{P \mid X \cap H_{1}}\right)=H^{2} \quad \text { and } \quad c_{1}\left(\mathcal{N}_{P \mid X \cap H_{1}}\right)=-H .
$$

Finally, since $P \subset \mathbb{P}^{4}=F \cap H_{1} \subset X \cap H_{1}$, consider the exact sequence

$$
0 \rightarrow \mathcal{N}_{P \mid \mathbb{P}^{4}} \cong \mathcal{O}_{\mathbb{P}^{3}}(1) \rightarrow \mathcal{N}_{P \mid X \cap H_{1}} \rightarrow \mathcal{N}_{\mathbb{P}^{4}\left|X \cap H_{1}\right| P} \rightarrow 0 .
$$

Put $C_{i}:=c_{i}\left(\mathcal{N}_{\mathbb{P}^{4}\left|X \cap H_{1}\right| P}\right)$ for $i=1,2$. Thus we get the system

$$
\left\{\begin{array}{l}
C_{1}+H=-H \\
C_{2}+C_{1} H=H^{2} \\
C_{2} H=-H^{3}
\end{array}\right.
$$

but its resolution gives a numerical contradiction.

So we conclude that for any $n \geq 2 m-1$ with $m \leq 4$, all fibers of $p$ are of dimension $n-m$. Therefore by $[15,(2.12)] p$ is a $\mathbb{P}^{d}$-bundle over the smooth manifold $Y$ with $d=n-m \geq m-1$ and $m \leq 4$.

In particular, as consequences of Theorem 2.2, we get the following

Corollary 2.3. Let $(X, L)$ be an n-dimensional scroll, $p: X \rightarrow Y$, over a normal projective variety $Y$ of dimension $m \geq 4$ and let $L$ be a very ample line bundle on $X$. Let $\mathcal{Z}:=\left\{y \in Y \mid \operatorname{dim}^{-1}(y)>n-m\right\}$. If the general fiber of $p$ has dimension bigger or equal to 3 , then $\operatorname{cod}_{Y} \mathcal{Z} \geq 5$.

Proof. By slicing with general hyperplane sections on $Y$ we can assume that $m=4$ and $\operatorname{dim} X=n-m+4$. Hence $\mathcal{Z}=\emptyset$ by Theorem 2.2.

Proposition 2.4. Let $X$ be a smooth projective $(2 m-2)$-fold, $L$ an ample line bundle on it. Assume that $(X, L)$ is an adjunction-theoretic scroll over a $m$-fold $Y$ with $3 \leq m \leq 4$ and let $\phi: X \rightarrow Y$ be the scroll projection. If $X \in|\mathcal{L}|$ where $\mathcal{L}$ is a very ample line bundle on a smooth $(2 m-1)$-fold $M$, and the restriction of $\mathcal{L}$ to $X$ is $L$, then $M$ is a $\mathbb{P}^{m-1}$-bundle on $Y$ and $\phi: X \rightarrow Y$ either is a $\mathbb{P}^{m-2}$-bundle on $Y$ or it has some special fibers isomorphic to $\mathbb{P}^{m-1}$. In particular, $\phi$ has no divisorial fibers. 
Proof. We have

$$
\left(K_{M}+m \mathcal{L}\right)_{X} \simeq K_{X}+(m-1) L \simeq \phi^{*} H
$$

for some ample line bundle $H$ on $Y$. Then by [28, (2.1)] $K_{M}+m \mathcal{L}$ is nef and some positive power of it gives a morphism $\Phi: M \rightarrow \mathbb{P}^{N}$. Note that by the reasoning in the proof of $[28,(0.3 .2)]$ one has $\Phi(M)=Y$ and $(\delta)$ becomes

$$
\left(K_{M}+m \mathcal{L}\right)_{X} \simeq \phi^{*} H \simeq\left(\Phi^{*} H\right)_{X}
$$

Then $\left(K_{M}+m \mathcal{L}\right) \simeq \Phi^{*} H$ and $(M, \mathcal{L})$ is an adjunction-theoretic scroll over $Y$ under $\Phi$. Since $\operatorname{dim} M=2 m-1=2 \operatorname{dim} Y-1$, from Theorem 2.2 it follows that $M$ is a $\mathbb{P}^{m-1}$-bundle over $Y$. Therefore the dimension of any fiber of $\Phi$, and hence of $\phi$, is bounded by $m-1<2 m-3=\operatorname{dim} X-1$ and so by [15, (2.12)] and [6, (4.1)(ii)] we obtain the statement.

\section{Classical scrolls}

Let $X$ be a smooth $n$-fold with $n \geq 4$ and let $L$ be an ample line bundle on $X$. Assume that $(X, L)$ is a $\mathbb{P}^{k}$-bundle, $\pi: X \rightarrow Y$, over a smooth variety $Y$ of dimension $m$. Then $(X, L) \cong\left(\mathbb{P}(\mathcal{E}), \mathcal{O}_{\mathbb{P}(\mathcal{E})}(1)\right)$, where $\mathcal{E}=\pi_{*} L$ is an ample vector bundle over $Y$ of rank $k+1$. We know that for $n \geq 2 m-1$ the polarized pair $(X, L)$ is also an adjunction-theoretic scroll over $Y$ except for a few exceptions (see [11, (2.1)]). As to the case $n=2 m-2$, i.e., $k=m-2$, we give here the following immediate consequence of [23], [11, (3.1)] and [1, Theorem].

Proposition 3.1. Let $X$ be a smooth $n$-fold and let $L$ be an ample line bundle on $X$. Assume that $(X, L) \cong\left(\mathbb{P}(\mathcal{E}), \mathcal{O}_{\mathbb{P}(\mathcal{E})}(1)\right)$ is a $\mathbb{P}^{n-m}$-bundle, $\pi: X \rightarrow Y$, over a smooth variety $Y$ of dimension $m \geq 3$ with $\mathcal{E}=\pi_{*} L$. If $n=2 m-2$, then $(X, L)$ is an adjunction-theoretic scroll over $Y$ under $\pi$ unless either:

(a) $Y \cong \mathbb{P}^{m}$ and $\mathcal{E} \cong \mathcal{O}_{\mathbb{P}^{m}}(1)^{\oplus m-1}, \mathcal{O}_{\mathbb{P} m}(2) \oplus \mathcal{O}_{\mathbb{P}^{m}}(1)^{\oplus m-2}$, $\mathcal{O}_{\mathbb{P} m}(2)^{\oplus 2} \oplus \mathcal{O}_{\mathbb{P} m}(1)^{\oplus m-3}, \mathcal{O}_{\mathbb{P}^{m}}(3) \oplus \mathcal{O}_{\mathbb{P} m}(1)^{\oplus m-2} ;$

(b) $Y \cong \mathbb{P}^{3}$ and $\mathcal{E}$ is isomorphic to the twist $\mathcal{N}(2)$ of a null-correlation bundle $\mathcal{N}$ on $\mathbb{P}^{3}$

(c) $Y \cong \mathbb{Q}^{m}$ and $\mathcal{E}$ is either $\mathcal{O}_{\mathbb{Q}^{m}}(1)^{\oplus m-1}$ or $\mathcal{O}_{\mathbb{Q}^{m}}(2) \oplus \mathcal{O}_{\mathbb{Q}^{m}}(1)^{\oplus m-2}$;

(d) $Y \cong \mathbb{Q}^{4}$ and $\mathcal{E} \cong \mathcal{S}(2) \oplus \mathcal{O}_{\mathbb{Q}^{4}}(1)$, where $\mathcal{S}$ is a spinor bundle on $\mathbb{Q}^{4} \subset \mathbb{P}^{5}$;

(e) $Y \cong \mathbb{Q}^{3}$ and $\mathcal{E} \cong \mathcal{S}(2)$, where $\mathcal{S}$ is a spinor bundle on $\mathbb{Q}^{3} \subset \mathbb{P}^{4}$;

(f) $Y \cong \mathbb{P}^{2} \times \mathbb{P}^{1}$ and $\mathcal{E}$ is either $\mathcal{O}_{Y}(2,1) \oplus \mathcal{O}_{Y}(1,1)$ or $p_{1}^{*}\left(T_{\mathbb{P}^{2}}\right) \otimes \mathcal{O}_{Y}(0,1)$, where $p_{1}: Y \rightarrow \mathbb{P}^{2}$ is the projection of $Y$ onto the first factor;

(g) $Y \cong \mathbb{P}^{2} \times \mathbb{P}^{2}$ and $\mathcal{E} \cong \mathcal{O}_{\mathbb{P}^{2} \times \mathbb{P}^{2}}(1,1)^{\oplus 3}$;

(h) $Y$ is a Del Pezzo 3-fold with $b_{2}(Y) \geq 2$ such that $-K_{Y} \simeq(m-1) H$ for an ample line bundle $H$ on $Y$ and $\mathcal{E} \cong H^{\oplus 2}$;

(i) $Y$ is a Del Pezzo $m$-fold with $b_{2}(Y)=1$, i.e., $\operatorname{Pic}(Y)$ is generated by an ample line bundle $\mathcal{O}_{Y}(1)$ such that $-K_{Y} \simeq \mathcal{O}_{Y}(m-1)$ and $\mathcal{E} \cong \mathcal{O}_{Y}(1)^{\oplus m-1}$;

(1) there is a vector bundle $\mathcal{V}$ on a smooth curve $C$ such that $Y \cong \mathbb{P}_{C}(\mathcal{V})$ and $\mathcal{E}_{F} \cong$ $\mathcal{O}_{F}(1)^{\oplus m-1}$ for any fiber $F \cong \mathbb{P}^{m-1}$ of $Y \rightarrow C$; 
(m) $m \geq 4$ and there is a surjective morphism $f: Y \rightarrow C$ onto a smooth curve $C$ such that any general fiber $F$ of $f$ is a smooth hyperquadric $\mathbb{Q}^{m-1}$ in $\mathbb{P}^{m}$ with $\mathcal{E}_{F} \cong \mathcal{O}_{F}(1)^{\oplus m-1}$

(n) $m \geq 4$ and there is a vector bundle $\mathcal{V}$ on a smooth surface $S$ such that $Y \cong \mathbb{P}_{S}(\mathcal{V})$ and $\mathcal{E}_{F} \cong \mathcal{O}_{F}(1)^{\oplus m-1}$ for any fiber $F \cong \mathbb{P}^{m-2}$ of $Y \rightarrow S$;

(o) $m=3$ and $(X, L),(Y$, det $\mathcal{E})$ are Del Pezzo fibrations, $\varphi: X \rightarrow C, \alpha: Y \rightarrow C$, over a smooth curve $C$ with $\varphi=\alpha \circ p$. Let $\Delta, D$ be the general fibers of $\varphi, \alpha$ respectively. Then either

- $D \cong \mathbb{P}^{2}$ and either $\Delta \cong \mathbb{P}\left(\mathcal{O}_{\mathbb{P}^{2}}(2) \oplus \mathcal{O}_{\mathbb{P}^{2}}(1)\right)$ or $\Delta \cong \mathbb{P}\left(T_{\mathbb{P}^{2}}\right)$, or

- $D \cong \mathbb{P}^{1} \times \mathbb{P}^{1}$ and $\Delta \cong \mathbb{P}^{1} \times \mathbb{P}^{1} \times \mathbb{P}^{1}$;

(p) $m=3,(X, L)$ is a quadric fibration $\varphi: X \rightarrow S$ over a smooth surface $S$ and $X$ is the fiber product $Y \times_{S} Y^{\prime}$ where $Y, Y^{\prime}$ are both $\mathbb{P}^{1}$-bundle $\alpha: Y \rightarrow S, \alpha^{\prime}: Y^{\prime} \rightarrow S$ over $S$ in the complex topology. Furthermore, $K_{Y}+\operatorname{det} \mathcal{E} \cong \alpha^{*} H$ for some ample line bundle $H$ on $S$;

(q) there exists a smooth projective $m$-fold $W$ and a morphism $\pi: Y \rightarrow W$ expressing $Y$ as blown up at a finite set $B$ of points and an ample vector bundle $\mathcal{E}^{\prime}$ on $W$ such that $\mathcal{E}=\pi^{*} \mathcal{E}^{\prime} \otimes\left[-\pi^{-1}(B)\right]$ and $K_{W}+\operatorname{det} \mathcal{E}^{\prime}$ is ample.

In particular, for $n \geq 6$ and $m=4$, we deduce from Proposition 3.1 the following

Corollary 3.2. Let $X$ be a smooth $n$-fold with $n \geq 6$ and let $L$ be an ample line bundle on $X$. Assume that $(X, L) \cong\left(\mathbb{P}(\mathcal{E}), \mathcal{O}_{\mathbb{P}(\mathcal{E})}(1)\right)$ is a $\mathbb{P}^{n-4}$-bundle, $\pi: X \rightarrow Y$, over a smooth 4-fold $Y, \mathcal{E}=\pi_{*} L$. Then $(X, L)$ is an adjunction-theoretic scroll over $Y$ under $\pi$ unless either:

1. $n=8, Y \cong \mathbb{P}^{4}$ and $\mathcal{E} \cong \mathcal{O}_{\mathbb{P}^{4}}(1)^{\oplus 5}$;

2. $n=7, Y \cong \mathbb{P}^{4}$ and $\mathcal{E} \cong \mathcal{O}_{\mathbb{P}^{4}}(1)^{\oplus 4}, \mathcal{O}_{\mathbb{P}^{4}}(2) \oplus \mathcal{O}_{\mathbb{P}^{4}}(1)^{\oplus 3}, T_{\mathbb{P}^{4}}$;

3. $n=7, Y \cong \mathbb{Q}^{4}$ and $\mathcal{E} \cong \mathcal{O}_{\mathbb{Q}^{4}}(1)^{\oplus 4}$;

4. $n=7$ and there is a vector bundle $\mathcal{V}$ over a smooth curve $C$ such that $Y \cong \mathbb{P}_{C}(\mathcal{V})$ and $\mathcal{E}_{F} \cong \mathcal{O}_{F}(1)^{\oplus 4}$ for any fiber $F \cong \mathbb{P}^{3}$ of $Y \rightarrow C$;

5. $n=6, Y \cong \mathbb{P}^{4}$ and $\mathcal{E} \cong \mathcal{O}_{\mathbb{P}^{4}}(1)^{\oplus 3}, \mathcal{O}_{\mathbb{P}^{4}}(2) \oplus \mathcal{O}_{\mathbb{P}^{4}}(1)^{\oplus 2}, \mathcal{O}_{\mathbb{P}^{4}}(2)^{\oplus 2} \oplus \mathcal{O}_{\mathbb{P}^{4}}(1)$, $\mathcal{O}_{\mathbb{P}^{4}}(3) \oplus \mathcal{O}_{\mathbb{P} 4}(1)^{\oplus 2}$

6. $n=6, Y \cong \mathbb{Q}^{4}$ and $\mathcal{E} \cong \mathcal{O}_{\mathbb{Q}^{4}}(1)^{\oplus 3}, \mathcal{O}_{\mathbb{Q}^{4}}(2) \oplus \mathcal{O}_{\mathbb{Q}^{4}}(1)^{\oplus 2}, \mathcal{E} \cong \mathcal{S}(2) \oplus \mathcal{O}_{\mathbb{Q}^{4}}(1)$, where $\mathcal{S}$ is a spinor bundle on $\mathbb{Q}^{4} \subset \mathbb{P}^{5}$;

7. $n=6$, $Y$ is a Del Pezzo 4-fold with $-K_{Y} \cong 3 H$ and $\mathcal{E} \cong H^{\oplus 3}$;

8. $n=6$ and there is a vector bundle $\mathcal{V}$ on a smooth curve $C$ such that $Y \cong \mathbb{P}_{C}(\mathcal{V})$ and $\mathcal{E}_{F} \cong \mathcal{O}_{F}(1)^{\oplus 3}$ for any fiber $F \cong \mathbb{P}^{3}$ of $Y \rightarrow C$;

9. $n=6$ and there is a surjective morphism $f: Y \rightarrow C$ onto a smooth curve $C$ such that any general fiber $F$ of $f$ is a smooth hyperquadric $\mathbb{Q}^{3}$ in $\mathbb{P}^{4}$ with $\mathcal{E}_{F} \cong \mathcal{O}_{F}(1)^{\oplus 3}$;

10. $n=6$ and there is a vector bundle $\mathcal{V}$ on a smooth surface $S$ such that $Y \cong \mathbb{P}_{S}(\mathcal{V})$ and $\mathcal{E}_{F} \cong \mathcal{O}_{F}(1)^{\oplus 3}$ for any fiber $F \cong \mathbb{P}^{2}$ of $Y \rightarrow S$;

11. $n=6$ and there exists a smooth projective 4-fold $W$ and a morphism $\pi: Y \rightarrow W$ expressing $Y$ as blow up at a finite set $B$ of points and an ample vector bundle $\mathcal{E}^{\prime}$ on $W$ such that $\mathcal{E} \cong \pi^{*} \mathcal{E}^{\prime} \otimes\left[-\pi^{-1}(B)\right]$ and $K_{W}+\operatorname{det} \mathcal{E}^{\prime}$ is ample. 
Proof. If $n \geq 9$, then $k=n-4 \geq 5>\operatorname{dim} Y$ and so $(X, L)$ is an adjunction-theoretic scroll over $Y$ by [11, (2.1.1)]. If $6 \leq n \leq 8$, then we can conclude by [11, (2.1.2), (2.1.3)] and Proposition 3.1.

Remark 3.3. Using [2, (5.1)], we can obtain satisfactory results similar to Proposition 3.1 and Corollary 3.2 also for the cases $n=2 m-3, m \geq 5$ and $n \geq 7, m=5$ respectively.

Therefore, for $\mathbb{P}^{n-4}$-bundles, $\pi: X \rightarrow Y$, over smooth varieties $Y$ of dimensions $m=4$ with $n=\operatorname{dim} X \geq 5$, it remains to consider the case $n=5$. Note that [2, (5.1)] does not cover completely this situation since there the results work well only for $m \geq 5$.

On the other hand, in line with the proof of $[11,(3.1)]$ and together with $[4$, Proposition 6], and [24, (1.3)] we finally deduce the following result for $n=5$ and $m=4$.

Proposition 3.4. Let $X$ be a smooth 5-fold and let $L$ be an ample line bundle on $X$. Assume that $(X, L) \cong\left(\mathbb{P}(\mathcal{E}), \mathcal{O}_{\mathbb{P}(\mathcal{E})}(1)\right)$ is a $\mathbb{P}^{1}$-bundle, $\pi: X \rightarrow Y$, over a smooth 4-fold $Y, \mathcal{E}=\pi_{*} L$. Then $(X, L)$ is an adjunction-theoretic scroll over $Y$ under $\pi$ unless either:

1. $Y \cong \mathbb{P}^{4}$ and $\mathcal{E} \cong \mathcal{O}_{\mathbb{P}^{4}}(1)^{\oplus 2}, \mathcal{O}_{\mathbb{P}^{4}}(2) \oplus \mathcal{O}_{\mathbb{P}^{4}}(1), \mathcal{O}_{\mathbb{P}^{4}}(3) \oplus \mathcal{O}_{\mathbb{P}^{4}}(1), \mathcal{O}_{\mathbb{P}^{4}}(2)^{\oplus 2}$;

2. $Y \cong \mathbb{Q}^{4}$ and $\mathcal{E} \cong \mathcal{O}_{\mathbb{Q}^{4}}(1)^{\oplus 2}, \mathcal{O}_{\mathbb{Q}^{4}}(2) \oplus \mathcal{O}_{\mathbb{Q}^{4}}(1), \mathcal{S} \otimes \mathcal{O}_{\mathbb{Q}^{4}}(2)$, where $\mathcal{S}$ is a spinor bundle on $\mathbb{Q}^{4}$;

3. $Y$ is a Del Pezzo 4-fold with $\operatorname{Pic}(Y) \cong \mathbb{Z}[H],-K_{Y}=3 H$ and $\mathcal{E} \cong H^{\oplus 2}$;

4. there is a vector bundle $\mathcal{V}$ on a smooth curve $C$ such that $Y \cong \mathbb{P}_{C}(\mathcal{V})$ with projection $p: \mathbb{P}_{C}(\mathcal{V}) \rightarrow C$ and either

$(\alpha) \mathcal{E} \cong \xi_{\mathcal{V}} \otimes p^{*} \mathcal{G}$ with $\mathcal{E}_{F} \cong \mathcal{O}_{\mathbb{P}^{3}}(1)^{\oplus 2}$ for any fiber $F \cong \mathbb{P}^{3}$ of $p$, where $\mathcal{G}$ is an ample vector bundle of rank two on $C$ and $\xi_{\mathcal{V}}$ is the tautological line bundle on $Y$, or

( $\beta$ ) there exists an exact sequence $0 \rightarrow p^{*} \mathcal{L} \otimes \xi_{\mathcal{V}}^{\otimes 2} \rightarrow \mathcal{E} \rightarrow p^{*} \mathcal{H} \otimes \xi_{\mathcal{V}} \rightarrow 0$ with $\mathcal{E}_{F} \cong \mathcal{O}_{\mathbb{P}^{3}}(2) \oplus \mathcal{O}_{\mathbb{P}^{3}}(1)$ for any fiber $F \cong \mathbb{P}^{3}$ of $p$, where $\mathcal{L}$ and $\mathcal{H}$ are line bundles on $C$;

5. there exists a hyperquadric fibration $q: Y \rightarrow C$ of the relative Picard number one over a smooth curve $C$, a q-ample line bundle $\mathcal{O}_{Y}(1)$ on $Y$ and an ample vector bundle $\mathcal{G}$ of rank two on $C$ such that $\mathcal{E} \cong \mathcal{O}_{Y}(1) \otimes q^{*} \mathcal{G}$ with $\mathcal{E}_{F} \cong \mathcal{O}_{\mathbb{Q}^{3}}(1)^{\oplus 2}$ for any fiber $F \cong \mathbb{Q}^{3} \subset \mathbb{P}^{4}$ of $q$;

6. there is a vector bundle $\mathcal{V}$ on a smooth surface $S$ such that $Y \cong \mathbb{P}_{S}(\mathcal{V})$ and $\mathcal{E}_{F} \cong$ $\mathcal{O}_{\mathbb{P}^{2}}(1)^{\oplus 2}$ for any fiber $F \cong \mathbb{P}^{2}$ of $Y \rightarrow S$;

7. $Y$ is a Fano 4-fold, $K_{X} \simeq-2 L$ and $(X, L)$ is a ruled Fano 5-fold of index two over $Y$ (see [22] for a detailed description of these pairs);

8. $(X, L),(Y, \operatorname{det} \mathcal{E})$ are Mukai fibrations, $g: X \rightarrow C, f: Y \rightarrow C$, over a smooth curve $C$ and $g=f \circ \pi$; moreover, if $F_{g}$ and $F_{f}$ are general fibers of $g$ and $f$ respectively, then one of the following possibilities can occur:

(a) $F_{f} \cong \mathbb{P}^{3}$ and $F_{g} \cong \mathbb{P}(\mathcal{N}(2))$, where $\mathcal{N}$ is the null-correlation bundle on $\mathbb{P}^{3}$;

(b) $F_{f} \cong \mathbb{P}^{3}$ and either $F_{g} \cong \mathbb{P}\left(\mathcal{O}_{\mathbb{P}^{3}}(2)^{\oplus 2}\right)$ or $F_{g} \cong \mathbb{P}\left(\mathcal{O}_{\mathbb{P}^{3}}(1) \oplus \mathcal{O}_{\mathbb{P}^{3}}(3)\right)$;

(c) $F_{f} \cong \mathbb{Q}^{3}$ and either $F_{g} \cong \mathbb{P}\left(\mathcal{O}_{\mathbb{Q}^{3}}(1) \oplus \mathcal{O}_{\mathbb{Q}^{3}}(2)\right)$ or $F_{g} \cong \mathbb{P}(\mathcal{S}(2))$, where $\mathcal{S}$ is the spinor bundle on $\mathbb{Q}^{3} \subset \mathbb{P}^{4}$;

(d) $F_{f} \cong \mathbb{P}^{2} \times \mathbb{P}^{1}$ and either $F_{g} \cong \mathbb{P}^{1} \times \mathbb{P}\left(T_{\mathbb{P}^{2}}\right)$ or $F_{g} \cong \mathbb{P}^{1} \times \mathbb{P}\left(\mathcal{O}_{\mathbb{P}^{2}}(1) \oplus \mathcal{O}_{\mathbb{P}^{2}}(2)\right)$; 
(e) $F_{f}$ is a Del Pezzo 3-fold, i.e., $-K_{F_{f}}=2 H$ for an ample line bundle $H$ on $F_{f}$ and $F_{g} \cong \mathbb{P}\left(H^{\oplus 2}\right) \cong \mathbb{P}^{1} \times F_{f}$;

9. $(X, L),(Y, \operatorname{det} \mathcal{E})$ are Del Pezzo fibrations, $g: X \rightarrow S, f: Y \rightarrow S$, over a normal surface $S$ and $g=f \circ \pi$; moreover, if $F_{g}$ and $F_{f}$ are general fibers of $g$ and $f$ respectively, then one of the following cases can occur:

(A) $F_{f} \cong \mathbb{P}^{2}$ and either $F_{g} \cong \mathbb{P}\left(\mathcal{O}_{\mathbb{P}^{2}}(1) \oplus \mathcal{O}_{\mathbb{P}^{2}}(2)\right)$ or $F_{g} \cong \mathbb{P}\left(T_{\mathbb{P}^{2}}\right)$;

(B) $F_{f} \cong \mathbb{P}^{1} \times \mathbb{P}^{1}, F_{g} \cong \mathbb{P}\left(\mathcal{O}_{\mathbb{P}^{1} \times \mathbb{P}^{1}}(1,1)^{\oplus 2}\right) \cong \mathbb{P}^{1} \times \mathbb{P}^{1} \times \mathbb{P}^{1}$;

moreover, in Case $(A)$ the surface $S$ is smooth and $f$ is a $\mathbb{P}^{2}$-bundle locally trivial in the complex topology, while in Case $(B)$ we have that $f=h \circ \varphi_{R}: Y \stackrel{\varphi_{R}}{\longrightarrow} Z \stackrel{h}{\longrightarrow} S$, where $\varphi_{R}$ is the contraction of an extremal ray $R$ such that either

$\left(\mathrm{B}_{1}\right)$ the map $\varphi_{R}$ is the blow-up of the smooth 4-fold $Z$ at a point $p$ and $l(R)=3$, where $l(R)$ is the length of the extremal ray $R, E=\varphi_{R}^{-1}(p) \cong \mathbb{P}^{3}$ is the exceptional divisor of $\varphi_{R}$ with $E_{E}=\mathcal{O}_{\mathbb{P}^{3}}(-1)$ and $\mathcal{E}_{E} \cong \mathcal{O}_{\mathbb{P}^{3}}(2) \oplus \mathcal{O}_{\mathbb{P}^{3}}(1)$, or

$\left(\mathrm{B}_{2}\right) l(R)=2$ and, if $\mathcal{R}$ is the locus of $R$ and $\Delta$ is a general fiber of the restriction $\varphi_{R \mid \mathcal{R}}: \mathcal{R} \rightarrow \varphi_{R}(\mathcal{R})$, then one of the following possibilities holds:

$\left(\mathrm{B}_{2} ; 1\right) \mathcal{R}=Y,\left(\Delta, \mathcal{E}_{\Delta}\right) \cong\left(\mathbb{P}^{2}, \mathcal{O}_{\mathbb{P}^{2}}(1)^{\oplus 2}\right)$ and $Z$ is a projective variety with at most isolated rational and Gorenstein singularities;

$\left(\mathrm{B}_{2} ; 2\right) \mathcal{R}=Y,\left(\Delta, \mathcal{E}_{\Delta}\right) \cong\left(\mathbb{Q}^{2}, \mathcal{O}_{\mathbb{Q}^{2}}(1)^{\oplus 2}\right)$ and $Z$ is a smooth surface;

$\left(\mathrm{B}_{2} ; 3\right)$ the contraction $\varphi_{R}$ is divisorial and the triplet $\left(\mathcal{R},[\mathcal{R}]_{\mathcal{R}}, \mathcal{E}_{\mathcal{R}}\right)$ is either $\left(\mathbb{P}^{3}, \mathcal{O}_{\mathbb{P}^{3}}(-2), \mathcal{O}_{\mathbb{P}^{3}}(1)^{\oplus 2}\right)$ or $\left(\mathbb{Q}^{3}, \mathcal{O}_{\mathbb{Q}^{3}}(-1), \mathcal{O}_{\mathbb{Q}^{3}}(1)^{\oplus 2}\right)$, where $\mathbb{Q}^{3} \subset$ $\mathbb{P}^{4}$ is a possibly singular hyperquadric in $\mathbb{P}^{4}$;

$\left(\mathrm{B}_{2} ; 4\right)$ the contraction $\varphi_{R}$ is the blowing-up along a smooth curve $\varphi_{R}(\mathcal{R})$ on the smooth 4-fold $Z$ such that for all fibers $\Delta \subset \mathcal{R}$ we have that $\left(\Delta, \mathcal{E}_{\Delta}\right) \cong\left(\mathbb{P}^{2}, \mathcal{O}_{\mathbb{P}^{2}}(1)^{\oplus 2}\right) ;$

10. $(X, L),(Y, \operatorname{det} \mathcal{E})$ are quadric fibrations, $g: X \rightarrow V, f: Y \rightarrow V$, over a normal 3-fold $V$ and $g=f \circ \pi$; moreover, $f$ factors through a contraction $\rho$ of an extremal ray $R$ such that $l(R)=2$, where $l(R)$ is the length of $R$, and if $\mathcal{R}$ is the locus of $R$ and $\Delta$ is a general fiber of the restriction $\rho_{\mathcal{R}}: \mathcal{R} \rightarrow \rho(\mathcal{R})$, then $\mathcal{R}=Y$ and $\operatorname{dim} \Delta=1$, or

11. $(X, L)$ is a scroll, $g: X \rightarrow Y^{\prime}$, over a normal 4-fold $Y^{\prime}$ and a high multiple of $K_{Y}+\operatorname{det} \mathcal{E}$ defines a birational map, $f: Y \rightarrow Y^{\prime}$, which contracts an extremal face; let $R_{i}$, for $i$ in a finite set of indices, be the extremal rays spanning this face; call $\rho_{i}: Y \rightarrow W$ the contraction associated to one of the $R_{i}$. Then each $\rho_{i}$ is birational and divisorial; if $D$ is one of the exceptional divisors (we drop the index) and $B=\rho(D)$, we have that $\operatorname{dim} B \leq 1$ and either

(i) $f: Y \rightarrow Y^{\prime}$ is the blowing-up of $Y^{\prime}$ at a point and $\mathcal{E}$ fits into the following exact sequence $0 \rightarrow f^{*} \mathcal{E}^{\prime} \otimes \mathcal{O}_{Y^{\prime}}(-2 D) \rightarrow \mathcal{E} \rightarrow \mathcal{O}_{Y^{\prime}}(-D) \rightarrow 0$, where $\mathcal{E}^{\prime}$ is a vector bundle of rank two on $Y^{\prime}, D \cong \mathbb{P}^{3}$ is the exceptional divisor of $f$ and $\mathcal{E}_{D} \cong \mathcal{O}_{\mathbb{P}^{3}}(2) \oplus \mathcal{O}_{\mathbb{P}^{3}}(1)$,

or one of the following possibilities can occur:

(ii) $\operatorname{dim} B=0, D \cong \mathbb{P}^{3}, D_{\mid D} \simeq \mathcal{O}_{\mathbb{P}^{3}}(-1)$ and $\mathcal{E}_{D} \cong \mathcal{O}_{\mathbb{P}^{3}}(1)^{\oplus 2}$;

(iii) $\operatorname{dim} B=0, D \cong \mathbb{P}^{3}, D_{\mid D} \simeq \mathcal{O}_{\mathbb{P}^{3}}(-2)$ and $\mathcal{E}_{D} \cong \mathcal{O}_{\mathbb{P}^{3}}(1)^{\oplus 2}$;

(iv) $\operatorname{dim} B=0, D$ is a (possibly singular) quadric $\mathbb{Q}^{3}, D_{\mid D} \simeq \mathcal{O}_{\mathbb{Q}^{3}}(-1)$ and $\mathcal{E}_{D} \cong \mathcal{O}_{\mathbb{Q}^{3}}(1)^{\oplus 2}$ 
(v) $\operatorname{dim} B=1, W$ and $B$ are smooth projective varieties, $\rho$ is the blow-up of $W$ along $B$ and $\mathcal{E}_{f} \cong \mathcal{O}_{\mathbb{P}^{2}}(1)^{\oplus 2}$ for any fiber $f \cong \mathbb{P}^{2}$ of $\rho_{\mid D}: D \rightarrow B$.

Proof. If $K_{Y}+\operatorname{det} \mathcal{E}$ is not nef, then by [2, (5.1)] we obtain pairs $(Y, \mathcal{E})$ as from Cases 1 to 6 and Case 11 (ii) of the statement.

Assume now that $K_{Y}+\operatorname{det} \mathcal{E}$ is nef but not ample. Then there exists a curve $C$ on $Y$ such that $\left(K_{Y}+\operatorname{det} \mathcal{E}\right) \cdot C=0$ and this shows that the nef value $\tau$ of the pair $(Y, \mathcal{E})$, i.e., the minimum of the set of real numbers $t$ such that $K_{Y}+t \operatorname{det} \mathcal{E}$ is nef, is equal to one. Moreover, by the Kawamata-Shokurov Base point free Theorem, we have that $m\left(K_{Y}+\operatorname{det} \mathcal{E}\right)$ is spanned for some integer $m \geq 0$. Consider the relative morphism $f$ defined by $m\left(K_{Y}+\operatorname{det} \mathcal{E}\right)$. By taking $m>>0$, we can assume that $f: Y \rightarrow V$ is a morphism with connected fibers and normal image $V=f(Y)$ of dimension $k:=$ $\operatorname{dim} V \leq 4$. Moreover, $K_{Y}+\operatorname{det} \mathcal{E} \simeq f^{*} D$ for some ample line bundle $D$ on $V$. Let $g=f \circ \pi$, where $\pi: X=\mathbb{P}(\mathcal{E}) \rightarrow Y$ is the projection onto $Y$. Note that a general fiber $F_{g}$ of $g$ is isomorphic to $\mathbb{P}\left(\mathcal{E}_{F_{f}}\right)$ for any general fiber $F_{f}$ of $f$.

Let us proceed with a case-by-case analysis.

Let $k=0$. Then $K_{Y}+\operatorname{det} \mathcal{E}=\mathcal{O}_{Y}$ and by the projection formula we have that $K_{X}+2 L=\mathcal{O}_{X}$. Thus $(X, L)$ is a ruled Fano 5-fold of index two over a Fano 4-fold $Y$ (see [22]). This gives Case 7 in the statement.

Let $k=1$. Then $V$ is smooth curve and $f$ is flat. Moreover, for a general fiber $F_{f}$ of $f$, since $\operatorname{dim} F_{f}=3$, from [26, (0.3) and (0.4)] we deduce that $\left(F_{f}, \mathcal{E}_{F_{f}}\right)$ is one of the following pairs: (1) $\left(\mathbb{P}^{3}, \mathcal{N}(2)\right)$, where $\mathcal{N}$ is the null-correlation bundle on $\mathbb{P}^{3}$; (2) $\left(\mathbb{P}^{3}, \mathcal{O}_{\mathbb{P}^{3}}(2)^{\oplus 2}\right) ;(3)\left(\mathbb{P}^{3}, \mathcal{O}_{\mathbb{P}^{3}}(1) \oplus \mathcal{O}_{\mathbb{P}^{3}}(3)\right) ;(4)\left(\mathbb{Q}^{3}, \mathcal{S}(2)\right)$, where $\mathcal{S}$ is the spinor bundle on $\mathbb{Q}^{3} \subset \mathbb{P}^{4} ;(5)\left(\mathbb{Q}^{3}, \mathcal{O}_{\mathbb{Q}^{3}}(1) \oplus \mathcal{O}_{\mathbb{Q}^{3}}(2)\right) ;(6)\left(\mathbb{P}^{2} \times \mathbb{P}^{1}, p r_{1}^{*}\left(T_{\mathbb{P}^{2}}\right) \otimes \mathcal{O}_{\mathbb{P}^{2} \times \mathbb{P}^{1}}(0,1)\right)$, where $p r_{1}: \mathbb{P}^{2} \times \mathbb{P}^{1} \rightarrow \mathbb{P}^{2}$ is the projection onto the first factor; (7) $\left(\mathbb{P}^{2} \times \mathbb{P}^{1}, \mathcal{O}_{\mathbb{P}^{2}} \times \mathbb{P}^{1}(2,1) \oplus\right.$ $\left.\mathcal{O}_{\mathbb{P}^{2} \times \mathbb{P}^{1}}(1,1)\right) ;(8) F_{f}$ is a Del Pezzo 3-fold with $b_{2}\left(F_{f}\right)=1$, that is, $\operatorname{Pic}\left(F_{f}\right)$ is generated by an ample line bundle $\mathcal{O}_{F_{f}}(1)$ such that $-K_{F_{f}} \simeq 2 \mathcal{O}_{F_{f}}(1)$ and $\mathcal{E}_{F_{f}} \cong \mathcal{O}_{F_{f}}(1)^{\oplus 2} ;$ (9) $F_{f}$ is a Del Pezzo 3-fold with $b_{2}\left(F_{f}\right) \geq 2$ and $\mathcal{E}_{F_{f}} \cong H^{\oplus 2}$, where $-K_{F_{f}} \simeq 2 H$ for an ample line bundle $H$ on $F_{f}$. We only note that Cases (6) and (7) give $F_{g} \cong \mathbb{P}^{1} \times \mathbb{P}\left(T_{\mathbb{P}^{2}}\right)$ and $F_{g} \cong \mathbb{P}^{1} \times \mathbb{P}\left(\mathcal{O}_{\mathbb{P}^{2}}(1) \oplus \mathcal{O}_{\mathbb{P}^{2}}(2)\right)$ respectively (see [30]). This gives Case 8 in the statement.

Let $k=2$. Then the general fiber $F_{f}$ of $f$ is a surface and since $K_{F_{f}}+\operatorname{det} \mathcal{E}_{F_{f}} \simeq$ $\mathcal{O}_{F_{f}}$, we see that $\left(F_{f}, \operatorname{det} \mathcal{E}_{F_{f}}\right)$ is a Del Pezzo surface. Moreover, by arguing as in [11, p. 67] we obtain that $F_{f}$ is isomorphic to either $\mathbb{P}^{2}$ or $\mathbb{P}^{1} \times \mathbb{P}^{1}$. Thus by [16] we get that $\left(F_{f}, \mathcal{E}_{F_{f}}\right)$ is one of the following pairs: $\left(\mathrm{a}^{\prime}\right)\left(\mathbb{P}^{2}, \mathcal{O}_{\mathbb{P}^{2}}(1) \oplus \mathcal{O}_{\mathbb{P}^{2}}(2)\right) ;\left(\mathrm{b}^{\prime}\right)\left(\mathbb{P}^{2}, T_{\mathbb{P}^{2}}\right) ;\left(\mathrm{c}^{\prime}\right)$ $\left(\mathbb{P}^{1} \times \mathbb{P}^{1}, \mathcal{O}_{\mathbb{P}^{1}} \times \mathbb{P}^{1}(1,1)\right)$. Note that $f$ factorizes as $f=h \circ \varphi_{R}: Y \stackrel{\varphi_{R}}{\longrightarrow} Z \stackrel{h}{\longrightarrow} S$, where $\varphi_{R}$ is the contraction of an extremal ray $R$. From [29, (2.4)] we know that $l(R) \leq 3$. Since $-K_{Y} \cdot C=\operatorname{det} \mathcal{E} \cdot C=\operatorname{deg} \mathcal{E}_{C} \geq 2$ for any curve $C$ such that $[C] \in R$, we conclude that $2 \leq l(R) \leq 3$. Suppose that $F_{f} \cong \mathbb{P}^{2}$. Since for any curve $l \in\left|\mathcal{O}_{\mathbb{P}^{2}}(a)\right|$, $a \geq 1$, we have

$$
-K_{Y} l=-K_{F_{f}} l=\mathcal{O}_{\mathbb{P}^{2}}(3) \mathcal{O}_{\mathbb{P}^{2}}(a)=3 a \geq 3>\frac{1}{2}(\operatorname{dim} Y+1),
$$

by $[2,(1.12)]$ we obtain that $f$ is an elementary contraction, i.e., $f=\varphi_{R}$, and from [4, Propostion 2] it follows that $f$ is equidimensional and $Z \cong S$ is smooth. Using a 
similar argument as in $[1,(2.2)]$ we see that $f$ is a $\mathbb{P}^{2}$-bundle locally trivial in the complex topology over a smooth surface $S$. This leads to Case 9 (A) of the statement. Assume now that $F_{f} \cong \mathbb{P}^{1} \times \mathbb{P}^{1}$. If $l(R)=3$, then $\varphi_{R}$ cannot be of fiber type. Otherwise, by [4, Propositions 1 and 2] we have that $Z$ is a smooth surface and the general fiber of $\varphi_{R}$ is a $\mathbb{P}^{2}$. By arguing as in $[1,(2.2)]$ we deduce that any fiber of $\varphi_{R}$ is a $\mathbb{P}^{2}$, but this contradicts that the general fiber of $f=h \circ \varphi_{R}$, with $h$ birational, is a $\mathbb{P}^{1} \times \mathbb{P}^{1}$. Thus $\varphi_{R}$ is birational and [5, (1.1)] gives that $\varphi_{R}$ is the blow-up of $Z$ at a smooth point $p$ such that $E=\varphi_{R}^{-1}(p)$ is the exceptional divisor. Since $[E]_{E}=\mathcal{O}_{\mathbb{P}^{3}}(-1)$, we deduce that $c_{1}\left(\mathcal{E}_{E}\right)=\operatorname{det} \mathcal{E}_{E}=-K_{Y \mid E}=\mathcal{O}_{\mathbb{P}^{3}}(3)$, that is, $\mathcal{E}_{E} \cong \mathcal{O}_{\mathbb{P}^{3}}(2) \oplus \mathcal{O}_{\mathbb{P}^{3}}(1)$. This gives Case $9\left(\mathrm{~B}_{1}\right)$. Finally, if $l(R)=2$, by arguing as in [4, Proposition 6] we deduce all the possibilities of Case $9\left(\mathrm{~B}_{2}\right)$. This completes Case 9 of the statement.

Let now $k=3$. Then $(X, L)$ and $(Y, \operatorname{det} \mathcal{E})$ are quadric fibrations over a normal 3-fold $V$. From the Cone Theorem, we know that there exists an extremal ray, $R$, subordinate to $f$, i.e., such that $\left(K_{Y}+\operatorname{det} \mathcal{E}\right) \cdot R=0$. Let $\rho: Y \rightarrow Z$ be the contraction of $R$. Then $f$ factors through $\rho, f=\beta \circ \rho$. Let $\mathcal{R}$ be the locus of $R$ and let $\delta$ be a general fiber of the restriction $\rho_{\mathcal{R}}: \mathcal{R} \rightarrow \rho(\mathcal{R})$. By the Ionescu-Wiśniewski inequality (see [17, (0.4)] and $[31,(1.1)])$, we get

$$
2 \operatorname{dim} \mathcal{R} \geq \operatorname{dim} \mathcal{R}+\operatorname{dim} \delta \geq 4+l(R)-1 \geq 5,
$$

where $l(R)$ is the length of the ray $R$. Thus we have the following possibilities:

$$
\left(\mathrm{A}^{\prime}\right) \operatorname{dim} \mathcal{R}=4, \operatorname{dim} \delta=1 ; \quad\left(\mathrm{B}^{\prime}\right) \operatorname{dim} \mathcal{R}=\operatorname{dim} \delta=3 ; \quad\left(\mathrm{C}^{\prime}\right) \operatorname{dim} \mathcal{R}=3, \operatorname{dim} \delta=2 .
$$

Let us assume that $2 \leq \operatorname{dim} \delta \leq 3$. Clearly, $f(\delta)$ is a point, $v \in V$. Let $p$ be a point of $\delta$ such that $p$ does not belong to any other irreducible component of $f^{-1}(v)$. By taking a limit of general fibers of $f$, we can get a curve $C$ contained in $f^{-1}(v)$ and with $p \in C$. Note that $C$ is a union of rational curves since the general fiber $F_{f}$ of $f$ is a rational curve. Note also that $C$ is numerically equivalent to $F_{f}$ and therefore $\operatorname{det} \mathcal{E} \cdot C=\operatorname{det} \mathcal{E} \cdot F_{f}=-K_{Y} \cdot F_{f}=2$. Since $\mathcal{E}$ is an ample rank-2 vector bundle, it follows that $C$ is irreducible. Therefore $C$ must be contained in $\delta$ and hence $[C] \in R$. Then we have also $\left[F_{f}\right] \in R$ and this implies that $\mathcal{R}=Y$, but this is a contradiction. Thus only Case $\left(\mathrm{A}^{\prime}\right)$ can occur and this leads to Case 10 of the statement.

Finally, let $k=4$. Then $V$ is a normal 4-fold and $g: X \rightarrow V$ is an adjunctiontheoretic scroll. Recall that each fiber $F$ of $\pi$ is contained in fibers of $g$ since $m\left(K_{X}+2 L\right)$ is trivial on $F$. If $f$ is a finite morphism, since both $\pi$ and $g$ have connected fibers, we get a contradiction unless $\operatorname{deg} f=1$ which means that $f$ is an isomorphism. Thus in this case $(X, L)$ should be a scroll under $\pi$. Therefore we can assume that $f$ is a birational morphism, and since $f$ is given by $\left|m\left(K_{Y}+\operatorname{det} \mathcal{E}\right)\right|, m>>0$, we see that $K_{Y}+\operatorname{det} \mathcal{E}$ is nef and big but not ample. Thus we conclude as in [4, Proposition 6] (or [24, (1.3)]) obtaining Cases 11 (i), 11 (iii), 11 (iv) and 11 (v) of the statement.

\section{References}

[1] M. Andreatta, E. Ballico, J. Wiśniewski, Vector bundles and adjunction. Internat. J. Math. 3 (1992), 331-340. MR1163727 (93h:14031) Zbl 0770.14008 
[2] M. Andreatta, M. Mella, Contractions on a manifold polarized by an ample vector bundle. Trans. Amer. Math. Soc. 349 (1997), 4669-4683. MR1401760 (98b:14012) Zbl 0885.14004

[3] M. Andreatta, M. Mella, Morphisms of projective varieties from the viewpoint of minimal model theory. Dissertationes Math. (Rozprawy Mat.) 413 (2003), 1-72. Zbl 1080.14513

[4] M. Andreatta, C. Novelli, Manifolds polarized by vector bundles. Ann. Mat. Pura Appl. (4) 186 (2007), 281-288. MR2295120 (2008a:14056) Zbl pre05177470

[5] M. Andreatta, G. Occhetta, Special rays in the Mori cone of a projective variety. Nagoya Math. J. 168 (2002), 127-137. MR1942399 (2004d:14011) Zbl 1055.14015

[6] M. Andreatta, J. A. Wiśniewski, A note on nonvanishing and applications. Duke Math. J. 72 (1993), 739-755. MR1253623 (95c:14007) Zbl 0853.14003

[7] M. Andreatta, J. A. Wiśniewski, Contractions of smooth varieties. II. Computations and applications. Boll. Unione Mat. Ital. Sez. B Artic. Ric. Mat. (8) 1 (1998), 343-360. MR1638131 (99f:14021b) Zbl 1001.14003

[8] M. Andreatta, J. A. Wiśniewski, On contractions of smooth varieties. J. Algebraic Geom. 7 (1998), 253-312. MR1620110 (99f:14021a) Zbl 0966.14012

[9] M. C. Beltrametti, P. Ionescu, Erratum: "On manifolds swept out by high dimensional quadrics” [Math. Z. 260 (2008), no. 1, 229-234; MR2413352]. Math. Z. 260 (2008), 235-236. MR2413353 (2009g:14067b) Zbl 1146.14027

[10] M. C. Beltrametti, A. J. Sommese, New properties of special varieties arising from adjunction theory. J. Math. Soc. Japan 43 (1991), 381-412. MR1096439 (92d:14031) Zbl 0754.14027

[11] M. C. Beltrametti, A. J. Sommese, Comparing the classical and the adjunction-theoretic definition of scrolls. In: Geometry of complex projective varieties (Cetraro, 1990), volume 9 of Sem. Conf., 55-74, Mediterranean, Rende 1993. MR1225588 (94e:14053) Zbl 0937.14027

[12] M. C. Beltrametti, A. J. Sommese, The adjunction theory of complex projective varieties. De Gruyter 1995. MR1318687 (96f:14004) Zbl 0845.14003

[13] M. C. Beltrametti, A. J. Sommese, J. A. Wiśniewski, Results on varieties with many lines and their applications to adjunction theory. In: Complex algebraic varieties (Bayreuth, 1990), volume 1507 of Lecture Notes in Math., 16-38, Springer 1992. MR1178717 (93i:14007) Zbl 0777.14012

[14] L. Ein, Varieties with small dual varieties. II. Duke Math. J. 52 (1985), 895-907. MR816391 (87m:14048) Zbl 0603.14026

[15] T. Fujita, On polarized manifolds whose adjoint bundles are not semipositive. In: Algebraic geometry, Sendai, 1985, volume 10 of Adv. Stud. Pure Math., 167-178, North-Holland 1987. MR946238 (89d:14006) Zbl 0659.14002

[16] T. Fujita, On adjoint bundles of ample vector bundles. In: Complex algebraic varieties (Bayreuth, 1990), volume 1507 of Lecture Notes in Math., 105-112, Springer 1992. MR1178722 (93j:14052) Zbl 0782.14018

[17] P. Ionescu, Generalized adjunction and applications. Math. Proc. Cambridge Philos. Soc. 99 (1986), 457-472. MR830359 (87e:14031) Zbl 0619.14004

[18] Y. Kawamata, Small contractions of four-dimensional algebraic manifolds. Math. Ann. 284 (1989), 595-600. MR1006374 (91e:14039) Zbl 0661.14009

[19] Y. Kawamata, K. Matsuda, K. Matsuki, Introduction to the minimal model problem. In: Algebraic geometry, Sendai, 1985, volume 10 of Adv. Stud. Pure Math., 283-360, North-Holland 1987. MR946243 (89e:14015) Zbl 0672.14006 
[20] K. Matsuki, Introduction to the Mori program. Springer 2002. MR1875410 (2002m:14011) Zbl 0988.14007

[21] S. Mori, Threefolds whose canonical bundles are not numerically effective. Ann. of Math. (2) 116 (1982), 133-176. MR662120 (84e:14032) Zbl 0557.14021

[22] C. Novelli, G. Occhetta, Ruled Fano fivefolds of index two. Indiana Univ. Math. J. 56 (2007), 207-241. MR2305935 (2008e:14060) Zbl 1118.14048

[23] G. Occhetta, A note on the classification of Fano manifolds of middle index Manuscr. Math. 117 (2005), 43-49. Zbl 1083.14047

[24] M. Ohno, Classification of generalized polarized manifolds by their nef values. Adv. Geom. 6 (2006), 543-599. MR2267037 (2008b:14074a) Zbl 1138.14026

[25] C. Okonek, M. Schneider, H. Spindler, Vector bundles on complex projective spaces. Birkhäuser 1980. MR561910 (81b:14001) Zbl 0438.32016

[26] T. Peternell, M. Szurek, J. A. Wiśniewski, Fano manifolds and vector bundles. Math. Ann. 294 (1992), 151-165. MR1180456 (93h:14030) Zbl 0786.14027

[27] E. Sato, Projective manifolds swept out by large-dimensional linear spaces. Tohoku Math. J. (2) 49 (1997), 299-321. MR1464179 (98m:14046) Zbl 0917.14026

[28] A. J. Sommese, On the adjunction theoretic structure of projective varieties. In: Complex analysis and algebraic geometry (Göttingen, 1985), volume 1194 of Lecture Notes in Math., 175-213, Springer 1986. MR855885 (87m:14049) Zbl 0601.14029

[29] J. A. Wiśniewski, Length of extremal rays and generalized adjunction. Math. Z. 200 (1989), 409-427. MR978600 (91e:14032) Zbl 0668.14004

[30] J. A. Wiśniewski, Ruled Fano 4-folds of index 2. Proc. Amer. Math. Soc. 105 (1989), 55-61. MR929433 (89g:14032) Zbl 0687.14034

[31] J. A. Wiśniewski, On contractions of extremal rays of Fano manifolds. J. Reine Angew. Math. 417 (1991), 141-157. MR1103910 (92d:14032) Zbl 0721.14023

[32] J. A. Wiśniewski, On deformation of nef values. Duke Math. J. 64 (1991), 325-332. MR1136378 (93g:14012) Zbl 0773.14003

Received 9 November, 2007; revised 7 January, 2008

A. L. Tironi, Dipartimento di Matematica "F. Enriques", Via C. Saldini 50, 20133 Milano, Italy Email: atironi@mat.unimi.it 